\title{
Annealing-free Si3N4 frequency combs for monolithic integration with Si photonics
}

El Dirani, Houssein; Kamel, Ayman Nassar; Casale, Marco; Kerdiles, Sébastien; Monat, Christelle; Letartre, Xavier; Pu, Minhao; Oxenløwe, Leif Katsuo; Yvind, Kresten; Sciancalepore, Corrado

Published in:

Applied Physics Letters

Link to article, DOI:

$10.1063 / 1.5038795$

Publication date:

2018

Document Version

Publisher's PDF, also known as Version of record

Link back to DTU Orbit

Citation (APA):

El Dirani, H., Kamel, A. N., Casale, M., Kerdiles, S., Monat, C., Letartre, X., Pu, M., Oxenløwe, L. K., Yvind, K., \& Sciancalepore, C. (2018). Annealing-free Si3N4 frequency combs for monolithic integration with Si photonics. Applied Physics Letters, 113(8), [081102]. https://doi.org/10.1063/1.5038795

\section{General rights}

Copyright and moral rights for the publications made accessible in the public portal are retained by the authors and/or other copyright owners and it is a condition of accessing publications that users recognise and abide by the legal requirements associated with these rights.

- Users may download and print one copy of any publication from the public portal for the purpose of private study or research.

- You may not further distribute the material or use it for any profit-making activity or commercial gain

- You may freely distribute the URL identifying the publication in the public portal 


\section{Annealing-free $\mathrm{Si}_{3} \mathrm{~N}_{4}$ frequency combs for monolithic integration with Si photonics}

Houssein El Dirani, Ayman Kamel, Marco Casale, Sébastien Kerdiles, Christelle Monat, Xavier Letartre, Minhao Pu, Leif Katsuo Oxenløwe, Kresten Yvind, and Corrado Sciancalepore

Citation: Appl. Phys. Lett. 113, 081102 (2018); doi: 10.1063/1.5038795

View online: https://doi.org/10.1063/1.5038795

View Table of Contents: http://aip.scitation.org/toc/apl/113/8

Published by the American Institute of Physics

\section{Articles you may be interested in}

Thermal quenching of the yellow luminescence in GaN

Journal of Applied Physics 123, 161520 (2018); 10.1063/1.4995275

Guest Editorial: The dawn of gallium oxide microelectronics

Applied Physics Letters 112, 060401 (2018); 10.1063/1.5017845

Significant improvement in the electrical characteristics of Schottky barrier diodes on molecularly modified Gallium Nitride surfaces

Applied Physics Letters 112, 163502 (2018); 10.1063/1.5005587

\section{Conference Proceedings}




\title{
Annealing-free $\mathrm{Si}_{3} \mathrm{~N}_{4}$ frequency combs for monolithic integration with Si photonics
}

\author{
Houssein El Dirani, ${ }^{1, a)}$ Ayman Kamel, ${ }^{2}$ Marco Casale, ${ }^{1}$ Sébastien Kerdiles, ${ }^{1}$ \\ Christelle Monat, ${ }^{3}$ Xavier Letartre, ${ }^{3}$ Minhao Pu, ${ }^{2}$ Leif Katsuo Oxenløwe, ${ }^{2}$ Kresten Yvind, ${ }^{2}$ \\ and Corrado Sciancalepore ${ }^{1}$ \\ ${ }^{1}$ University Grenoble Alpes, CEA-LETI, Minatec, Optics and Photonics Division, 17 rue des Martyrs, \\ F-38054 Grenoble, France \\ ${ }^{2}$ DTU Fotonik, Technical University of Denmark, Kgs. DK-2800 Lyngby, Denmark \\ ${ }^{3}$ Institut des nanotechnologies de Lyon, UMR CNRS 5270, Ecole Centrale Lyon, F-69130 Ecully, France
}

(Received 5 May 2018; accepted 9 August 2018; published online 21 August 2018)

\begin{abstract}
Silicon-nitride-on-insulator (SiNOI) is an attractive platform for optical frequency comb generation in the telecommunication band because of the low two-photon absorption and free carrier induced nonlinear loss when compared with crystalline silicon. However, high-temperature annealing that has been used so far for demonstrating $\mathrm{Si}_{3} \mathrm{~N}_{4}$-based frequency combs made co-integration with silicon-based optoelectronics elusive, thus reducing dramatically its effective complementary metal oxide semiconductor (CMOS) compatibility. We report here on the fabrication and testing of annealing-free SiNOI nonlinear photonic circuits. In particular, we have developed a process to fabricate low-loss, annealing-free, and crack-free $\mathrm{Si}_{3} \mathrm{~N}_{4}$ 740-nm-thick films for Kerr-based nonlinear photonics featuring a full process compatibility with front-end silicon photonics. Experimental evidence shows that micro-resonators using such annealing-free silicon nitride films are capable of generating a frequency comb spanning $1300-2100 \mathrm{~nm}$ via optical parametrical oscillation based on four-wave mixing. This work constitutes a decisive step toward time-stable power-efficient Kerrbased broadband sources featuring full process compatibility with Si photonic integrated circuits on CMOS lines. Published by AIP Publishing. https://doi.org/10.1063/1.5038795
\end{abstract}

Kerr optical frequency combs constitute a paradigm shift in the development of high-capacity data transmission, integrated spectroscopy, high precision metrology, and frequency synthesis. ${ }^{1}$ Since 2010, silicon-nitride-on-insulator (SiNOI) has imposed as an attractive chip-based platform for the generation of wideband frequency combs pumped at telecom wavelengths because of its relatively high nonlinearity $(\times 10$ that of silica and larger than that of highly nonlinear

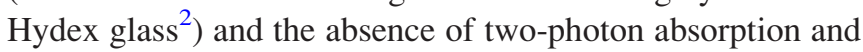
free carrier generation which plague crystalline silicon. In the meanwhile, silicon photonic integrated circuits (Si-PICs) have demonstrated increasing maturity levels for a wide range of optical functions such as III-V-on-Si integrated lasers, ${ }^{3}$ high-speed modulators, ${ }^{4}$ Ge-on-Si photodiodes, ${ }^{5}$ and filters and wavelength (de)multiplexers, ${ }^{6}$ thus continuously highlighting the potential of silicon optoelectronic integration with cost-effective complementary metal-oxide-semiconductor (CMOS) technology. ${ }^{7,8}$ In this context, the monolithic integration of Kerr-based frequency combs with Si photonics and CMOS micro-nano-electronic nodes holds the promise for on-chip high-capacity transmitters that would benefit from the maturity and low cost of CMOS manufacturing and scalability.

The realization of relatively thick $(>700 \mathrm{~nm})$ stoichiometric $\mathrm{Si}_{3} \mathrm{~N}_{4}$ films, as required by microring frequency combs, which imply both a tight confinement of light and anomalous group velocity dispersion (GVD), remains

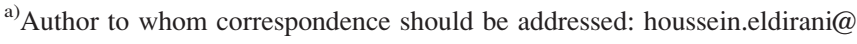
cea.fr
}

challenging. In particular, all prior works strictly made use of long high-temperature annealing $\left(\sim 1200^{\circ} \mathrm{C}\right.$ for at least 3 h) of the deposited silicon nitride film. ${ }^{9-12}$ This extreme annealing step has been accounted for by the need to densify the silicon nitride film through driving out excess hydrogen and break N-H bonds, so as to get closer to a stoichiometric $\mathrm{Si}_{3} \mathrm{~N}_{4}$ film and reduce the material absorption loss in the Cband. However, this annealing induces thermal stress that eventually leads to cracks during the device processing unless sophisticated pre-patterning strategies are adopted prior to the film deposition. ${ }^{10,11}$ Yet, in the perspective of nonlinear optics-silicon optoelectronic co-integration, these extreme annealing temperatures would severely degrade the front-end-of-the-line (FEOL) silicon optoelectronic circuits underneath. Specifically, doped optical circuits would be unacceptably affected by the undesirable dopant diffusion in junction-based $\mathrm{Si}$ modulators and by the hetero-interface degradation of Ge-on-Si photodetectors. As an alternative, optical parametric oscillation (OPO) has been demonstrated by using the proprietary composition of Hydex waveguides in $2013 .^{2}$ However, the nonlinear parameter $\gamma$ of Hydex is six times lower than that of silicon nitride, thus increasing the required power for a frequency comb generation, hence the risk of waveguide optical damage. Comb generation has also been demonstrated in 2018 using deuterated silicon nitride (SiN:D) deposited at $300{ }^{\circ} \mathrm{C}^{13}$ However, although being a CMOS-compatible processing building block, it is worth to point out that deuterated silicon nitride can be exclusively used during the back-end-of-the-line (BEOL) fabrication, thus preventing a straightforward monolithic 
integration with both silicon photonic and sub- $100 \mathrm{~nm}$ standard CMOS electronic circuitries during a collective FEOL processing. ${ }^{14}$ Furthermore, this material has a relatively strong thermal shift that occurs from the larger residual hydrogen-related absorption coefficient (when compared to purely stoichiometric $\mathrm{Si}_{3} \mathrm{~N}_{4}$ films), making it difficult to stabilize the comb in the soliton state as described in Ref. 13. In addition, for similar resonance quality factors, deuterated silicon nitride microresonators require higher threshold powers for comb generation than stoichiometric $\mathrm{Si}_{3} \mathrm{~N}_{4}$, which indicates a lower nonlinear index of SiN:D and increases, as for the Hydex, the risk of waveguide optical damage.

Very recently, we reported a method that avoids thermal annealing for growing relatively thick $(740 \mathrm{~nm})$ crack-free $\mathrm{Si}_{3} \mathrm{~N}_{4}$-based straight nanowaveguides with good linear and nonlinear properties, as measured by self-phase modulation of picosecond pulses propagating along these waveguides. ${ }^{15}$

In this paper, we report a comb generated from a microresonator made in an intrinsically CMOS-compatible annealingfree silicon nitride, following a tailored deposition method and without the typical hour-long $1200{ }^{\circ} \mathrm{C}$ post-annealing that is detrimental to the integration with front-end silicon optoelectronic circuits. Our annealing-free and crack-free fabrication process (shown in Fig. 1) provides our devices with the right specification (i.e., microring group velocity dispersion and characteristics) to underpin Kerr frequency combs, thus representing a significant step toward the full compatibility of $\mathrm{Si}_{3} \mathrm{~N}_{4}$-based Kerr comb sources with the thermal budgets of $\mathrm{Si}$ photonic processing. In contrast to all previous approaches, our process does not exceed neither the dopant activation temperature $\left(1030^{\circ} \mathrm{C}\right)$ required for $\mathrm{Si}$ modulators ${ }^{16}$ nor the $\mathrm{H}_{2}$ annealing thermal budget used for dislocation control of $\mathrm{Ge}-$ on-Si photodiodes $\left(825^{\circ} \mathrm{C}\right){ }^{17}$ Furthermore, our approach would offer a clear advantage even in the perspective of silicon photonic full integration with sub- $100 \mathrm{~nm}$ standard micronano-electronic CMOS circuits during collective FEOL processing. ${ }^{18}$

In order to control strain and to prevent cracks from appearing, the silicon nitride layer is deposited on a (non-patterned) substrate via low-pressure chemical vapor deposition (LPCVD) in two steps of 370-nm-thick layer each. The deposition is

$\begin{array}{ll}\text { (a) Substrate Thermal oxydation } & \text { (b) First Ultra-low SiN deposition }\end{array}$

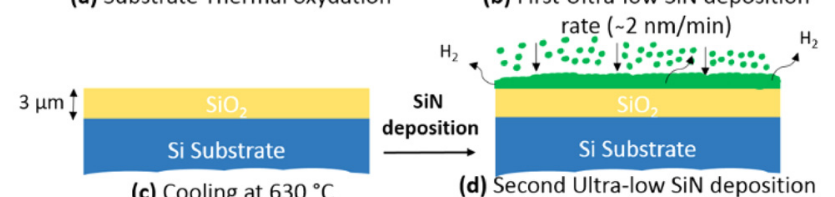

(c) Cooling at $630{ }^{\circ} \mathrm{C}$

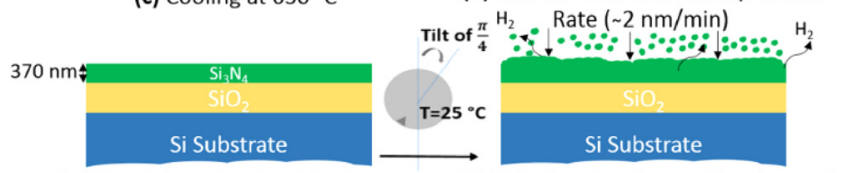

(e) Dry etch and resist stripping

(f) High-density plasma silica deposition

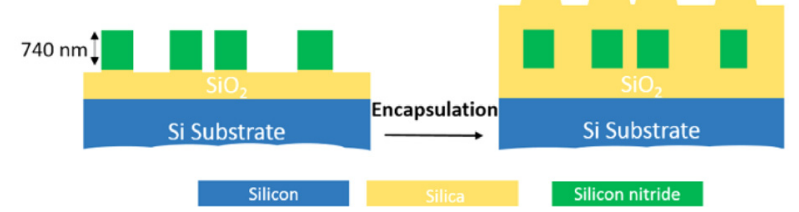

FIG. 1. Schematics of the annealing-free fabrication process for $\mathrm{Si}_{3} \mathrm{~N}_{4}$ nonlinear photonics (a)-(f). carried out with a tailored ultra-low deposition rate $(<2 \mathrm{~nm} /$ $\min$ ) to produce a very high quality film, which is denser optically and highly nonlinear $\left(n_{2}=3.6 \times 10^{-15} \mathrm{~cm}^{2} \mathrm{~W}^{-1}\right){ }^{15}$

Critically, under such a low deposition rate which is $40 \%$ lower than that of standard LPCVD silicon nitride reported in Ref. 19 and 30\% lower than the deposition rate mentioned in Ref. 20, the thermal activation energy enables silicon and nitrogen to dispose at the silicon nitride film surface via atomic surface migration phenomena while compelling hydrogen to escape the film. Furthermore, between the two deposition stages, the wafer is rotated by $45^{\circ}$ in order to distribute the uniaxial strain along the overall film thickness, thus avoiding film cracks upon subsequent subtractive patterning. Each deposition run is carried out at $780^{\circ} \mathrm{C}$ with post-deposition cooling to around $630^{\circ} \mathrm{C}$ for $20 \mathrm{~min}$. Controlled ramp-ups and ramp-downs from/to $780^{\circ} \mathrm{C}$ at $10^{\circ} \mathrm{C} / \mathrm{min}$ to/from $630^{\circ} \mathrm{C}$ are used prior to each deposition which is carried out under a pressure of 112 mTorr using $\mathrm{NH}_{3}(200 \mathrm{sccm})$ and $\mathrm{SiH}_{2} \mathrm{Cl}_{2}(80 \mathrm{sccm})$ precursor gases, thus providing a ratio between the two precursor gases $\left(\mathrm{SiH}_{2} \mathrm{Cl}_{2}: \mathrm{NH}_{3}\right)$ of 0.4. For comparison, the deposition of silicon-rich silicon nitride requires this ratio to be higher than $2,{ }^{21}$ which typically implies an increase in the $\mathrm{SiH}_{2} \mathrm{Cl}_{2}$ gas flow and a decrease in the $\mathrm{NH}_{3}$ gas flow rate. According to Ref. 22 , a decrease in the $\mathrm{NH}_{3}$ gas flow inevitably translates into an increase in the deposition rate. Regarding nitride-rich silicon nitride films, the deposition rate is of the order of $17 \mathrm{~nm} / \mathrm{min},{ }^{23}$ which is much higher than our deposition rate too.

The long thermal annealing step adopted in standard LPCVD processes is used to densify the SiN film, through bringing sufficient energy to break up N-H bonds, otherwise causing absorption in the material. By reducing the content of residual hydrogen ${ }^{11,19}$ in the SiN film, the high tensile strain is a clear indication of the film stoichiometry. ${ }^{19,21}$ In order to compare between the stoichiometry of films produced via our annealing-free process and by the standard LPCVD process including a long post-annealing step, we compared their respective tensile strains. The stress of our silicon nitride film is inferred by measuring the wafer bow, before and after removing the silicon nitride from the wafer back side. The material morphological characterization study revealed a tensile strain around $+1200 \mathrm{MPa}$, which is $1.5 x$ times higher than the value of $800 \mathrm{MPa}$ reported in Ref. 11, employing a long post-annealing step and considered, so far, as the state of the art in stoichiometric silicon nitride processing for nonlinear applications and optical parametric oscillators.

Another indication of the remaining N-H bonds is provided by the spectral dependence of the intrinsic quality factor $\left(Q_{i}\right)$ measured for a $\mathrm{Si}_{3} \mathrm{~N}_{4}$ micro-ring resonator with a waveguide width of $1.4 \mu \mathrm{m}$ (see Fig. 2). It roughly increases by a factor 2 while moving away from the N-H overtone absorption peak (near $1520 \mathrm{~nm}$ ), showing the presence of residual N-H bonds in our film. The losses in the ring can be estimated by the expression ${ }^{19} \alpha=\frac{k_{0} n_{g}}{Q_{i}}$, where $k_{0}$ is the wavenumber and $n_{g}$ is the mode group index of the waveguide with a similar width. The difference between the losses at $1550 \mathrm{~nm}\left(Q_{i}=350000\right)$ and the losses at $1520 \mathrm{~nm}$ $\left(Q_{i}=190000\right)$ can thus be estimated to be $0.9 \mathrm{~dB} / \mathrm{cm}$. This 


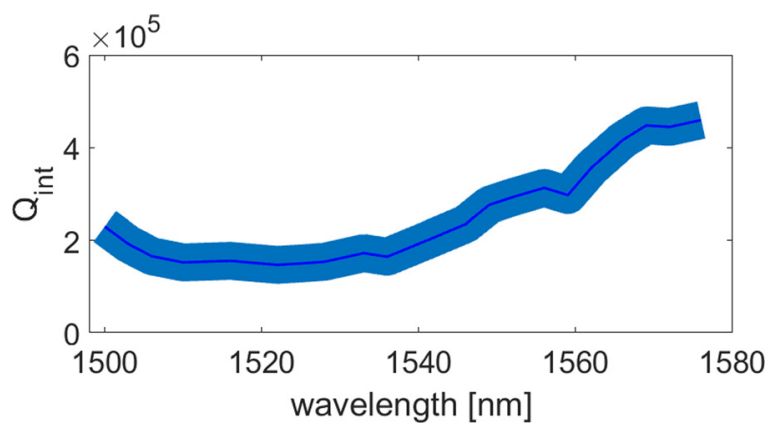

FIG. 2. Intrinsic quality factor as a function of the wavelength for an annealing-free silicon nitride microring resonator (waveguide width equal to $1.4 \mu \mathrm{m})$. The darker colored line shows the mean value, and the brighter shadowed areas illustrate the standard deviation of the measurements.

additional loss due to residual N-H absorption for our annealing-free process is comparable to the value $(0.6 \mathrm{~dB} /$ $\mathrm{cm}$ ) inferred for high-temperature annealed $\mathrm{Si}_{3} \mathrm{~N}_{4}$ waveguides ${ }^{21}$ and as shown below, it does not preclude the oscillation and comb generation in the C-band.

The $\mathrm{Si}_{3} \mathrm{~N}_{4}$ circuits were patterned through a combination of deep UV lithography (I-line, 780-nm-thick resist) and fluoride-based $\left(\mathrm{CF}_{4}-\mathrm{CH}_{2} \mathrm{~F}_{2}-\mathrm{O}_{2}\right)$ dry etching. They were encapsulated by a $3-\mu$ m-thick $\mathrm{SiO}_{2}$ cladding layer at $400{ }^{\circ} \mathrm{C}$ using high-density plasma-enhanced chemical vapor deposition (HDP-PECVD) to avoid void formation, which is considered to be one of the main causes for device failure. Figure 3(b) shows the optical and electron scanning microscopy images of the fabricated devices.

The combination between the four-wave mixing processes within the resonator and the optical parametric oscillation results in Kerr frequency comb generation. The optical spectrum out of an annealing-free silicon-nitride-on-insulator microring with a 56- $\mu$ m radius is shown in Fig. 3(a). The cross-sectional dimensions (1.5- $\mu$ m-wide $\times 740$-nm-thick $)$ of the ring waveguide ensure that GVD is anomalous at the $1569 \mathrm{~nm}$ pump wavelength. The intrinsic quality factor of the microring - which is separated by a $350 \mathrm{~nm}$ gap from the bus waveguide-exceeds 580000 . A relatively smooth native line spacing $(\sim 406 \mathrm{GHz})$ frequency comb spanning across about $800 \mathrm{~nm}$ between $1300 \mathrm{~nm}$ and $2100 \mathrm{~nm}$ is obtained for $\sim 1 \mathrm{~W}$ TE-polarized continuous-wave power $\left(P_{\text {in }}\right)$ coupled in the bus waveguide. This power value is comparable to the power required $(2 \mathrm{~W})$ to fill the entire comb span using high-temperature annealed $\mathrm{Si}_{3} \mathrm{~N}_{4}$ rings with a loaded quality factor of 100000 (intrinsic quality factor, $200000)^{2}$ and to the pump power $(3 \mathrm{~W}$ ) used to generate an optical frequency comb in similar annealed $\mathrm{Si}_{3} \mathrm{~N}_{4}$ ring resonators. ${ }^{24}$ A spectrum recorded for $P_{\text {in }} \sim 300 \mathrm{~mW}$, i.e., well above the oscillation threshold, is also shown in Fig. 3(c). The comb spacing is wider in this case, as dictated by the spectral gain of degenerate four-wave mixing imposed by the device GVD.

In order to draw a comparison between our annealingfree process and the standard LPCVD process using a long post-annealing, some wafers have been annealed at $1050^{\circ} \mathrm{C}$ in a $\mathrm{N}_{2}$ atmosphere for $2 \mathrm{~h}$. This annealing condition was the most extreme we could apply on our CMOS line. Quite surprisingly, we did not observe a clear and significant improvement in the resonance linewidths. One primary reason might be related to our relatively low annealing temperature value as compared with that used by other groups (typically $>1200^{\circ} \mathrm{C}$ ) which is very close to the silicon wafer fusion limit. ${ }^{12}$ A second explanation might originate from the significant value of the mode scattering loss induced by the substantial microring side-wall roughness. The latter has still room for improvement to well below $<1 \mathrm{~nm}$ RMS via sophisticated pattering strategies which are currently under investigation. Such large scattering loss contribution to the Q-factor of our microring resonances may thus hide the
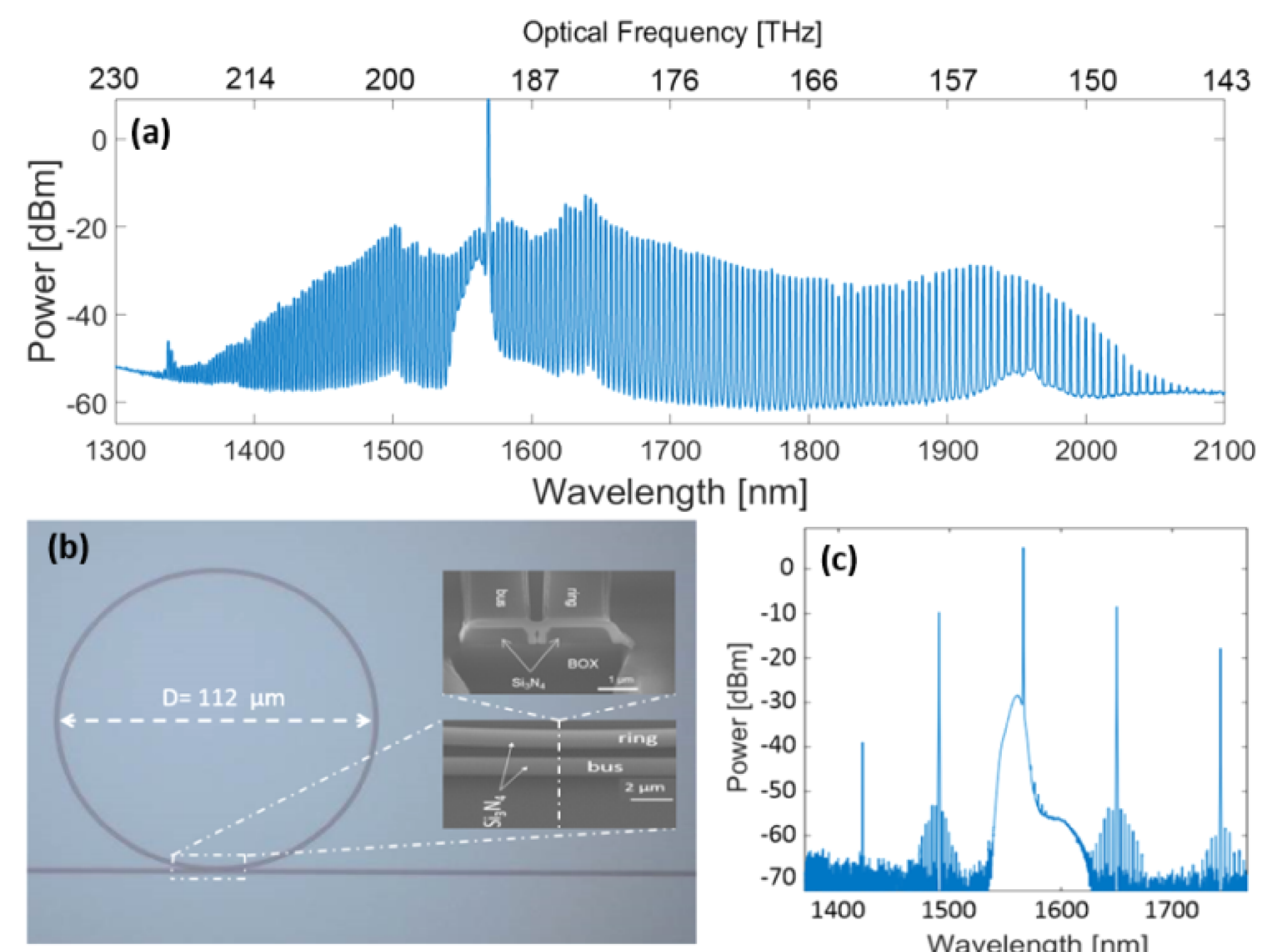

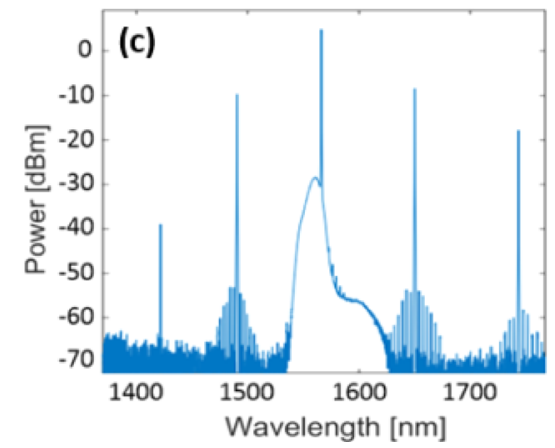

FIG. 3. Comb generation using annealing-free silicon nitride on insulator. (a) A 800-nm-spanning comb generation using a $56-\mu$ m-radius $\mathrm{Si}_{3} \mathrm{~N}_{4}$ microresonator for coupled power $P_{\text {in }}$ $\sim 1 \mathrm{~W}$. (b) Optical microscopy and (insets) scanning electron microscopy images of the ring and coupling region. (c) Comb spectrum for $P_{\text {in }} \sim 300 \mathrm{~mW}$. 
(a)

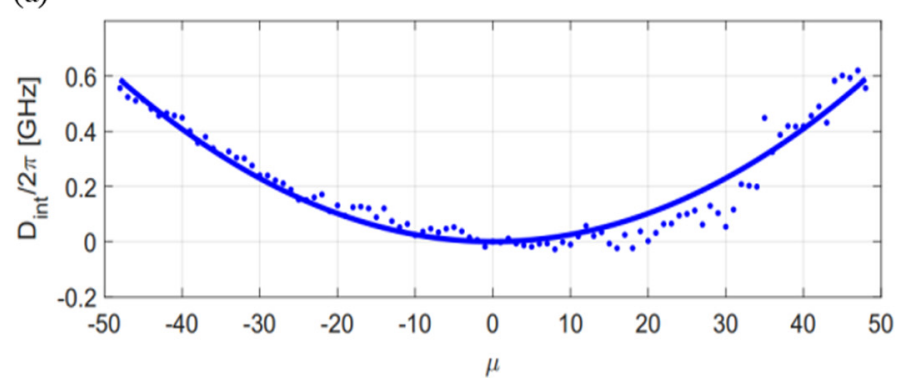

(b)

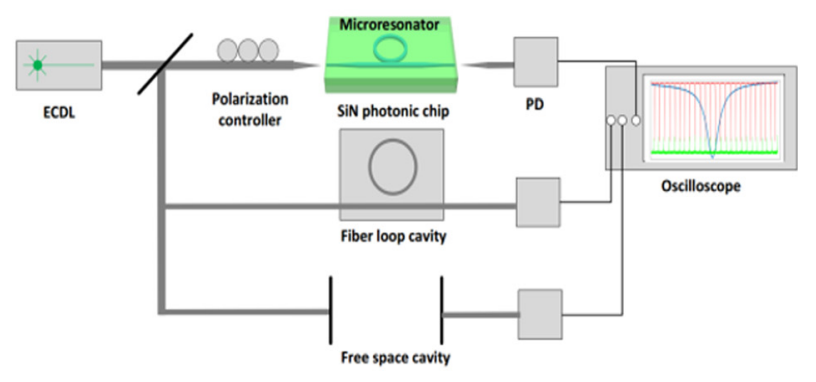

FIG. 4. Dispersion measurement of the TE mode of a ring resonator with a radius of $200 \mu \mathrm{m}$ and a width of $1.5 \mu \mathrm{m}$. (a) Plot of the integrated dispersion. (b) Experimental setup used for the dispersion measurement.

relatively minor gain in the $Q$ factor brought by the $1050^{\circ} \mathrm{C}$ annealing step, which likely falls within our $\mathrm{Q}$ factor measurement uncertainty. In any case, our work shows that a sufficient amount of hydrogen can be driven out of the $\mathrm{Si}_{3} \mathrm{~N}_{4}$ film using our deposition process, so as to get closer to stoichiometric silicon nitride films and to generate a frequency comb.

Regarding the damage threshold of our devices, throughout the whole measurement time ( $\sim 6$ months), we observed only one single waveguide fusion event, out of a total tested device number of $\sim 40$, which occurred at continuous-wave coupled power levels above $38 \mathrm{dBm}$. We think that such a fusion event originates from local waveguide imperfections (such a side-wall scattering or other local defects such as encapsulation voids and impurities) and waveguide ageing (notably at the upper cladding $\mathrm{Si}_{3} \mathrm{~N}_{4}-\mathrm{SiO}_{2}$ interface), partly due to the aforementioned residual N-H absorption tail in the annealing-free SiN films. Again though, this low device failure statistics points out that its impact is rather limited.

In order to measure the threshold power for optical parametric oscillation (OPO), the device was pumped with increasing power levels coupled to the bus waveguide. The laser wavelength was scanned across a resonance at $1570 \mathrm{~nm}$. The chip output light was passed through a short pass filter with a cut-off wavelength of $1560 \mathrm{~nm}$ which blocks the pump light with an extinction ratio of $60 \mathrm{~dB}$ and was then detected by a photodiode. The peak power of the filtered short wavelength OPO signal at each power coupled to the bus waveguide shows that the measured signal exceeds the noise floor (of $-50 \mathrm{dBm}$ ) above a threshold power of $\sim 83 \mathrm{~mW}$.

We measured the dispersion of our devices by scanning an external cavity diode laser (ECDL) light while recording the device transmission and two calibration traces from a fiber cavity and a high-finesse free space cavity with free spectrale range (FSR) values of $171 \mathrm{MHz}$ and $175 \mathrm{MHz}$, respectively. The integrated dispersion of the micro-cavity is $D_{\text {int }}(\mu)=\omega_{\mu}-D_{1} \mu=\frac{D_{2}}{2} \mu^{2}+\frac{D_{3}}{6} \mu^{3}+\cdots$, where $\omega_{\mu}$ is the angular frequency, $\mu$ is the mode number relative to the pumped mode, and $D_{i}$ are the dispersion coefficients. Figures 4(a) and 4(b) depict the measured dispersion and the measurement setup. A fit of the measured dispersion gives an anomalous dispersion value of $D_{2} / 2 \pi=511 \mathrm{kHz}$ at $1580 \mathrm{~nm}$ which is equivalent to a group velocity dispersion of $29 \mathrm{ps} /$ $\mathrm{nm} \mathrm{km}$. This value compares well with our finite element modeling of the dispersion which gave us an anomalous dispersion estimate of $50 \mathrm{ps} / \mathrm{nm} \mathrm{km}$ at the same wavelength. Note that the values of simulated and measured dispersion are in agreement with the simulated dispersion of similar annealed $\mathrm{Si}_{3} \mathrm{~N}_{4}$ waveguides $(1.65-\mu \mathrm{m}$-wide $\times 730$-nm-thick $)$ presented in Refs. 2 and 19.

In conclusion, these results show that it is possible to generate a wideband comb at telecom wavelengths using annealing-free silicon nitride nonlinear circuits featuring a full FEOL process compatibility with Si photonics. We demonstrated that annealing-free silicon nitride frequency comb microresonators can be made following a tailored deposition method, minimizing the hydrogen content. Our annealingfree and crack-free fabrication process provides our devices with the right specification (microring group velocity dispersion and characteristics) to underpin Kerr frequency combs, thus representing a significant step toward the full compatibility of $\mathrm{Si}_{3} \mathrm{~N}_{4}$-based Kerr-comb source monolithic integration with standard CMOS and $\mathrm{Si}$ photonic processing. Through allowing the monolithic integration of broadband comb sources with CMOS-compatible optoelectronics, our work represents a milestone toward the realization of nextgeneration Petabit/s data transmitters on a chip.

This work was supported by the DOPT 2020 internal funding program (CEA-LETI), the Danish Research Council SPOC (DNRF-123) center of excellence silicon photonics for communications (SPOC) (DTU), ERC H2020 GRAPHICS (648546), and the Institut Universitaire de France (INL).

${ }^{1}$ P. Marin-Palomo, J. N. Kemal, M. Kaprov, A. Kordts, J. Pfeifle, M. H. Pfeiffer, P. Trocha, S. Wolf, V. Brasch, M. H. Anderson, R. Rosenberger, K. Vijayan, W. Freude, T. J. Kippenberg, and C. Koos, Nature 546, 274 (2017).

${ }^{2}$ D. J. Moss, R. Morandotti, A. L. Gaeta, and M. Lipson, Nat. Photonics 7, 597 (2013).

${ }^{3}$ B. B. Bakir, A. Descos, N. Olivier, D. Bordel, P. Grosse, E. Augendre, L. Fulbert, and J.-M. Fedeli, Opt. Express 19, 10317 (2011).

${ }^{4}$ L. Liao, D. Samara-Rubio, M. Morse, A. Liu, D. Hodge, D. Rubin, U. Keil, and T. Franck, Opt. Express 13, 3129 (2005).

${ }^{5}$ L. Vivien, M. Rouvière, J.-M. Fedeli, D. Marris-Morini, J. F. Damlencourt, J. Mangeney, P. Crozat, L. E. Melhaoui, E. Cassan, X. Le Roux, D. Pascal, and S. Laval, Opt. Express 15, 9843 (2007).

${ }^{6}$ W. Bogaerts, S. K. Selvaraja, P. Dumon, J. Brouckaert, K. De Vos, D. Van Thourhout, and R. Baets, IEEE J. Sel. Top. Quantum Electron. 16, 33 (2010).

${ }^{7}$ G. T. Reed, Nature 427, 595-596 (2004).

${ }^{8}$ M. Asghari and A. V. Krishnamoorthy, Nat. Photonics 5, 268-270 (2011).

${ }^{9}$ J. Levy, A. Gondarenko, M. A. Foster, A. C. Turner-Foster, A. L. Gaeta, and M. Lipson, Nat. Photonics 4, 37 (2010). 
${ }^{10}$ K. Luke, A. Dutt, C. B. Poitras, and M. Lipson, Opt. Express 21, 22829 (2013).

${ }^{11}$ M. H. Pfeiffer, A. Kordts, V. Brasch, M. Zervas, M. Geiselmann, J. D. Jost, and T. J. Kippenberg, Optica 3, 20 (2016).

${ }^{12}$ S. Kim, K. Han, C. Wang, J. A. Jaramillo-Villegas, X. Xue, C. Bao, Y. Xuan, D. E. Leaird, A. M. Weiner, and M. Qi, Nat. Commun. 8, 372 (2017).

${ }^{13}$ J. Chiles, N. Nader, D. D. Hickstein, S. P. Yu, T. C. Briles, D. Carlson, H. Jung, J. M. Shainline, S. Diddams, S. B. Papp, S. W. Nam, and R. P. Mirin, Opt. Lett. 43, 1527 (2018).

${ }^{14}$ Y. A. Vlasov, IEEE Commun. Mag. 50(2), s67 (2012).

${ }^{15}$ H. El Dirani, M. Casale, S. Kerdiles, C. Socquet-Clerc, X. Letartre, C. Monat, and C. Sciancalepore, IEEE Photonics Technol. Lett. 30, 355 (2018).

${ }^{16}$ M. Streshinsky, R. Ding, Y. Liu, A. Novack, Y. Yang, Y. Ma, X. Tu, E. K. S. Chee, A. E. Lim, P. G. Lo, T. Baehr-Jones, and M. Hochberg, Opt. Express 21, 30350 (2013).
${ }^{17}$ J. Michel, J. Liu, and L. C. Kimerling, Nat. Photonics 4, 527 (2010).

${ }^{18}$ R. C. Johnson, see https://www.eetimes.com/document.asp?doc_id=1326576 for Electronic Engineering Times (2015).

${ }^{19}$ J. S. Levy, Ph.D. thesis, Cornell University, New York, 2011.

${ }^{20}$ J. Riemensberger, K. Hartinger, T. Herr, V. Brasch, R. Holzwarth, and T. J. Kippenberg, Opt. Express 20, 27661 (2012).

${ }^{21}$ C. J. Krückel, A. Fülöp, Z. Ye, P. A. Andrekson, and V. Torres-Company, Opt. Express 25, 15370 (2017).

${ }^{22}$ C. Yang and J. Pham, Silicon 1 (2018).

${ }^{23}$ L. Wang, H. S. Reehal, F. L. Martinez, E. S. Andres, and A. del Prado, Semicond. Sci. Technol. 18, 633 (2003).

${ }^{24}$ T. Herr, K. Hartinger, J. Riemensberger, C. Y. Wang, E. Gavartin, R. Holzwarth, M. L. Gorodetsky, and T. J. Kippenberg, Nat. Photonics 6, 480 (2012). 